Full length article

\title{
Long-term experience with a novel uterine-sparing transvaginal mesh procedure for uterovaginal prolapse
}

\author{
Giulio Nicita*, Donata Villari, Vincenzo Li Marzi, Martina Milanesi, Omar Saleh, \\ Tommaso Jaeger, Alberto Martini
}

Department of Urology, University of Florence, Careggi Hospital, Florence, Italy

\section{A R T I C L E I N F O}

\section{Article history:}

Received 26 February 2017

Received in revised form 3 November 2017

Accepted 8 January 2018

\section{Keywords:}

Mesh

Pelvic organ prolapse

Quality of life

Transvaginal surgery

Uterus sparing surgery

\begin{abstract}
A B S T R A C T
Objectives: To evaluate outcomes and quality of life in patients operated transvaginally with an original mesh shape for uterus-sparing prolapse surgery and to demonstrate the safety and efficacy of the technique.

Study design: We prospectively evaluated 66 postmenopausal patients (POP-Q Stage III: 32, IV: 34) operated between May 2008 and December 2013. We used wide weave polypropylene monofilament mesh that functions as a hammock anchored posteriorly to sacrospinous ligaments, its anterior wings exit the pelvis through the obturatory membrane.

Follow-up was scheduled at 3-, 12- months and in May 2016. Prolapse-Quality of Life Questionnaire (PQoL) was administered preoperatively, at 12 months and in May 2016. The chi square and Wilcoxon test were used for statistical analysis.

Results: Mean follow-up was 5.6 (SD: 1.6, Range: $1.1-8.1$ ) years. The overall success rate (POP-Q $\leq 2$ ) was $92.5 \%$ at 12 months and $84.4 \%$ at May 2016, these data remained stable over time $(\mathrm{p}>0.05)$. Early complications occurred in $2(3 \%)$ patients, late in $5(7.8 \%)$ of which mesh extrusion in 4 (6.3\%). Data from $\mathrm{P}-\mathrm{Q}$ oL showed significant improvement between preoperative and postoperative data $(\mathrm{P}<0.01$ for all domains) and they remained stable with time $(\mathrm{p}>0.05)$. De-novo dyspareunia was $17.6 \%$ at 12 months and $10.3 \%$ at May 2016.

Conclusions: The low rate and grade of complications demonstrates the safety of the procedure, which offers stable anatomical correction with significant improvement in QoL.
\end{abstract}

(c) 2018 Elsevier B.V. All rights reserved.

\section{Introduction}

Patients diagnosed with high-grade uterine prolapse, either III-IV according to the Pelvic Organ Prolapse Staging System (POP-Q), often require surgical treatment to correct their anatomofunctional defect. There are many surgical options, such as vaginal hysterectomy combined with native tissue repair including uterosacral or sacrospinous ligament suspension. Another option is to use synthetic materials in the repair or apical suspension. Further still, uterus-sparing surgery may be performed, again with various combinations and options for repair and suspension using native tissue and/or synthetic materials [1]. Recently the U.S. Food and Drug Administration (FDA) increased data requirements for the use of surgical meshes for pelvic organ prolapse (POP) repair through the vagina, and such meshes were re-categorized as class

\footnotetext{
* Corresponding author at: Clinica Urologica II, Azienda Ospedaliera Careggi, Università di Firenze, Viale San Luca, 50134, Firenze, Italy.

E-mail address: gnicita@unifi.it (G. Nicita).
}

III instead of class II which made them high-risk devices [2]. The FDA policy decision affected trans-vaginal procedures employing synthetic meshes, even outside the USA. Yet, is it realistic that many studies describing the anatomical rationale for the use of a mesh in POP and the thousands of patients who have successfully undergone this treatment have all been an error?

Our two decades' experience with mesh surgery prompts a critique of the FDA reclassification. We began to repair POP with mesh in the 1990s [3], and have persisted to the present because our results have been consistently more than satisfactory. In fact, we report only a modest rate of minor complications.

In our center we adopt two original vaginal techniques, one for single prolapse of the anterior segment (cystocele) [3], and the other for prolapse of the middle segment (cystocele and uterus or vaginal vault) [4]. For each defect we use soft, macroporous, wide weave polypropylene monofilament mesh (type 1 according to the Amid classification [5]) from the commercial market. Specifically, from 2006 we selected Gynemesh (Ethicon) and used it until 2013 though in 2012 it had gone off the market. Now we use Restorelle ${ }^{\circledR}$ 
Flat Mesh which we cut into two different shapes particular to the two types of prolapse. The novelties of our procedure are the shape of the mesh and the method of anchoring it to the uterus.

The aims of the current study were to evaluate outcomes and patient satisfaction in cases of uterine prolapse (POP-Q stage III/IV) treated with vaginal mesh, while preserving the uterus.

The primary objective of the study was to assess the effectiveness of anatomical correction, safety and complications. The secondary objective was to evaluate the resolution of symptoms and the effect on quality of life (QoL).

\section{Study design}

We performed a prospective observational study between May 2008 and December 2013. In average, at our center 20 patients receive procedure for anterior prolapse yearly, while 10-12 patients receive operation for POP yearly; $2-3$ patients are referred to the gynecology department either for patient's preference or for underlying gynecological pathologies such as uterine fibromatosis.
We enrolled 66 patients with high grade anterior and apical descensus, i.e. point $\mathrm{Aa}, \mathrm{Ba}$ and point $\mathrm{C}$ at stage $>2$ according to the POP-Q. Absolute exclusion criteria were: hypersensitivity to synthetic materials, previous hysterectomy or POP surgery, postmenopausal bleeding, abnormal cervical smears, uterine disease, an adnexal masses, or BMI $>30 \mathrm{~kg} / \mathrm{m} 2$.

For each patient the utility of a mesh implant was carefully evaluated. Informed consent, clarifying the novelty of the technique, the possibility of failure, and correlative risks was obtained from all participants.

The characteristics of the mesh are shown in Fig. 1. The mesh corrects the weakened fascial support that led to the POP by recreating the two levels of support for the bladder and the uterus theorized by DeLancey [6]. The dimensions conform to average pelvic diameters.

Preoperative patient assessment consisted of history, pelvic examination using POP-Q supine stress test with prolapse reduced, to detect occult stress incontinence that was measured using the Ingelman-Sundberg scale [7]. Urodynamic evaluation

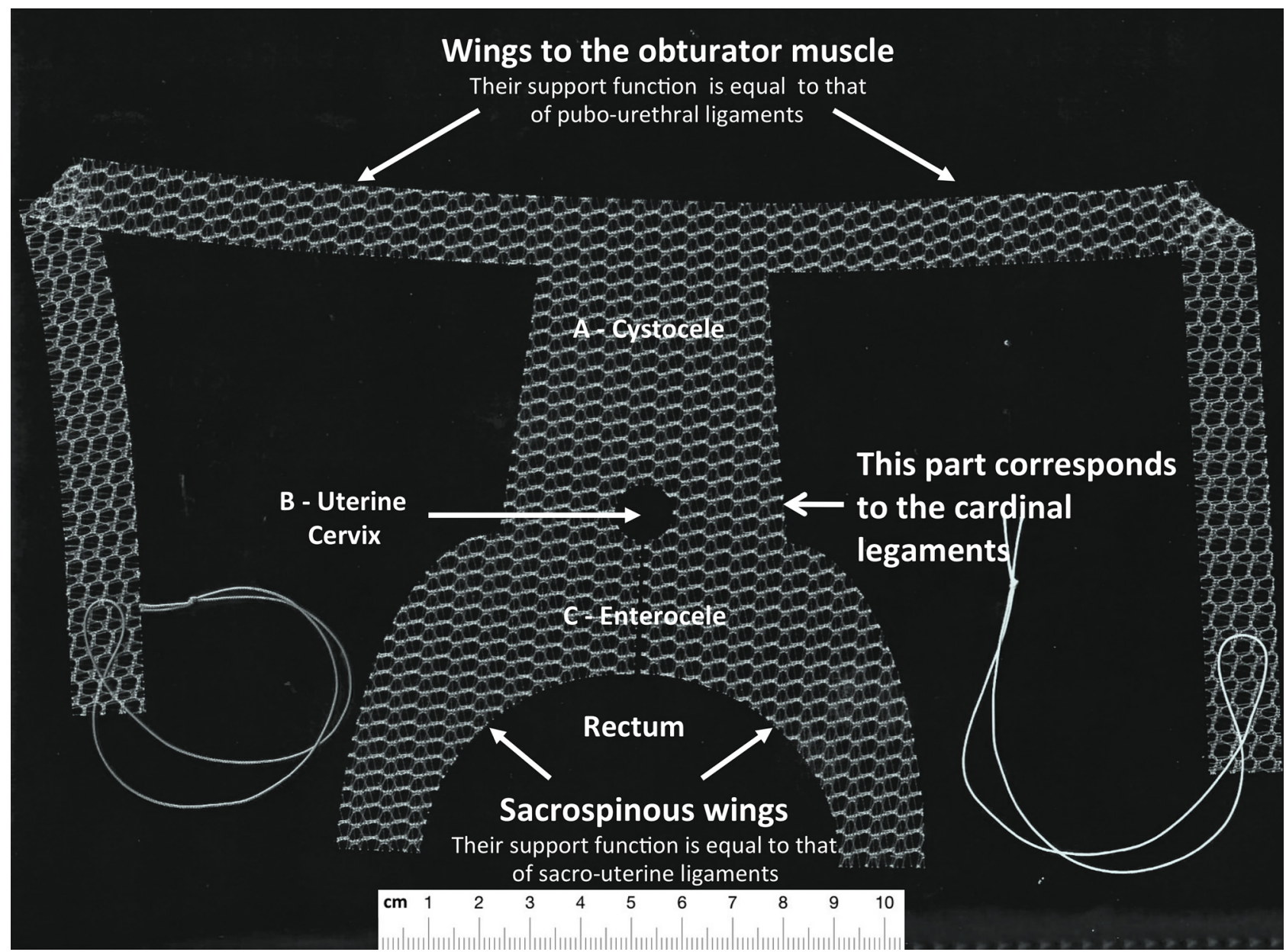

Fig. 1. The Rationale for the Shape of the Mesh.

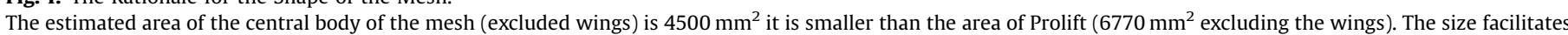

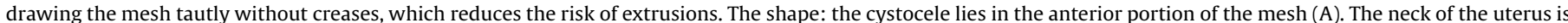

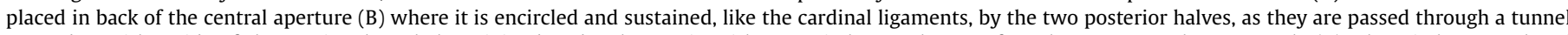

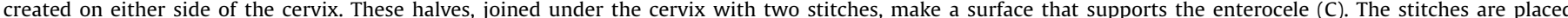

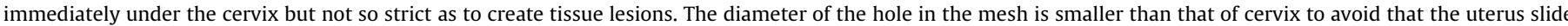

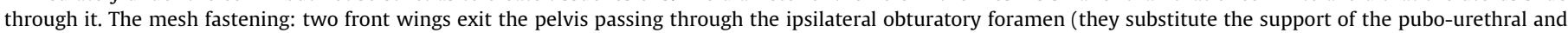

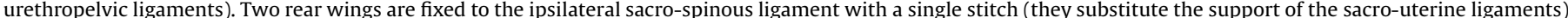

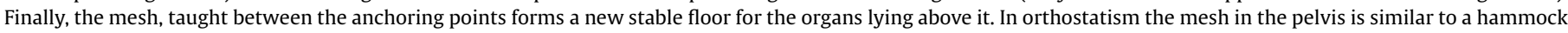
pulled tautly between the pubis and the sacrum. 
included uroflowmetry, post-void residual volume, filling cystometry and pressure/flow study with and without prolapse reduction using a pessary, following the International Continence Society (ICS) standard [8]. All patients were asked to compile the Italian version of the Prolapse-Quality of Life Questionnaire (P-QoL) [9].

All patients were post-menopausal. Baseline patients' characteristics, LUTS and pre-operative urodynamic data are listed in Table 1.

\section{Surgical technique}

All patients, after general anaesthesia, were placed in the dorsal lithotomy position and a $16 \mathrm{~F}$ bladder catheter was introduced. A Scott retractor was positioned and a midline longitudinal anterior colpotomy was made from the bladder neck to the cervix. The pubo-cervical fascia, that lies over the cystocele was exposed up to its insertion on the ischio-pubic branch at both the right and the left sides. Each front wing of the prosthesis, connected to an insideout transobturator handle, was passed through the obturator membrane and the skin, and it was pulled out until the mesh reached the bladder. A midline posterior colpotomy was made from the fornix to the end of the enterocele, and by using a Kellytype curved forceps, Fig. 2, a passage between the posterior and anterior vaginal segments was pierced as close as possible to the apex of the cervix. Each half of the mesh was passed through the ipsilateral passage until the cervix was placed at the center of the aperture in the mesh, to fasten it to the prosthesis. Two sutures joined the medial edges of the halves of the mesh to create a support plane for the enterocele. The rear wings were fixed to the ipsilateral sacrospinous ligaments with a stitch using the Capio ${ }^{\circledR}$ instrument and the uterus was pushed up into its correct position. Eventually the colpotomies were sutured. Figs. 3 and 4 show the location of the mesh in the pelvis. Patients with stress urinary incontinence (SUI) received a single incision transvaginal sling. All procedures were performed by an expert surgeon (G.N.).

\section{Postoperative follow-up and data analysis}

Post-operative assessment was planned at 1 (functional data not considered for the study purposes), 3 and 12 months. All patients were offered a visit free of charge in May 2016. Each time the visit included a pelvic examination. P-QoL questionnaires were distributed at 12 months and in May 2016. Postoperative urodynamic analysis was performed only when needed.

We defined objective anatomical cure at POP-Q stage less than 2 , satisfactory cure at POP-Q stage equal to 2 , while unsuccessful repair was POP-Q stages of 3 or 4 .

We used the Clavien-Dindo grade for surgical complications and the IUGA/ICS classification for mesh-related ones [10,11].

\section{Table 1}

Baseline patients' characteristics, lower urinary tract symptoms (LUTS) and preoperative urodynamic data.

\begin{tabular}{ll}
\hline Mean age (SD, Range), years & $69.7(7.7,50-87)$ \\
Mean BMI (SD), kg/m² & $24.9(5.2)$ \\
Median parity (IQR), n & $2(1-2)$ \\
Filling phase LUTS, n (\%) & $28(42.4)$ \\
Voiding phase LUTS, n (\%) & $15(22.7)$ \\
Mean Voided volume (SD), ml & $348.75(98.41)$ \\
Mean Maximal capacity (SD), ml & $375.74(75.48)$ \\
Mean Q max (SD), ml/min & $18.07(7.12)$ \\
Mean Q average (SD), ml/min & $11.4(4.94)$ \\
Mean PVR (SD), ml & $44.81(69.07)$ \\
Mean MUCP (SD), cmH $\mathrm{H}_{2} \mathrm{O}$ & $54.26(20.12)$
\end{tabular}

SD: standard deviation; IQR: interquartile range; PVR: post-void residual; MUCP: Maximum urethral closure pressure.
For statistical analysis, the chi-square test was used to compare data from POP-Q at different times, the Wilcoxon test was used for comparing the differences in P-QoL. Results were considered statistically significant when $\mathrm{p}<0.05$.

Methods, definitions and units conform to the standards recommended by the ICS [12].

No financial assistance was received from any company in the design or execution of this study.

\section{Results}

All patients were alive at 3 and 12 months follow-up. In May 2016, 64 (97\%) patients were examined, 2 (3\%) were lost at followup. The mean follow-up time was 5.6 (SD: 1.6, Range: 1.1-8.1) years. 21 (32\%) patients with stress incontinence received contemporary SUI surgery using a single incision transvaginal sling. Mean operating time was $82 \pm 20 \mathrm{~min}$. Bladder catheter was removed on the third postoperative day. Median hospital stay was 3 (Range 3-5) days. The 30-day Clavien complication rate was 3\%, due in both cases to grade III A complications: a pelvic hematoma in one patient and urinary retention requiring one week of selfcatheterization in another who underwent concomitant SUI surgery. Late complications occurred in a total of 5 (7.8\%) patients; specifically: $1(1.5 \%)$ grade II de novo urge incontinence (without urodynamic detrusor overactivity) treated with antimuscarinics and, 4 (6.3\%) grade III A small vaginal mesh extrusion (IUGA/ICS classification 1, T3, S1, a in all cases) treated with vaginal removal of the exposed portion of the mesh as an outpatient that did not require even local anaesthesia. No patient referred de novo subjective complaints related to bowel function.

Concerning the primary objective, the overall success rate (POP$\mathrm{Q} \leq 2$ ) was $92.5 \%$ at 12 months and $84.4 \%$ at May 2016. We registered $3(4,5 \%)$ stage IV cases at 3 months. We considered these cases as failures, not complications. In our experiences early recurrence of the prolapse is probably due to a technical mistake, loosening of the stitches, breakage of the mesh or of the anchorage structure. At 12 months after surgery 42 (63.6\%) patients were cured: ( 9 (13.6\%) stage 0,33 (50\%) stage I) and 19 (28.9\%) presented satisfactory prolapse cure. 5 (7.6\%) were considered unsuccessful (2 (3\%) stage III and 3 (4.5\%) stage IV). These values tended to remain stable over time, Table 2, in fact no statistical difference was observed comparing postoperative data between 3 and 12 months $(\mathrm{p}>0.05)$ nor between 12 months and data collected in May 2016 ( $\mathrm{p}>0.05)$. In May 2016, 10 (15.6\%) patients of 64 compliant with follow-up, presented unsuccessful repair (2 of them underwent hysterectomy in another hospital while 5 stage III, 3 stage IV did not receive other procedures), rendering an overall success rate of $84.4 \%$.

Regarding the secondary objective, a statistical difference emerged between data from preoperative P-QoL questionnaires and from postoperative questioning ( $\mathrm{p}<0.01$ for all domains). However, a comparison of follow-up data for patients in May 2016 with their 12 months postoperative data showed no statistical difference ( $p>0.05$ for all domains), Fig. 5. No patients developed de novo SUI. Concerning the personal relationships domain of the P-QoL questionnaire, 32 patients were sexually inactive, not for reasons attributable to $\mathrm{POP}$ (either for age, lacking of partner, ecc.). Among the remaining 34 patients, at 12 months 11 (32.4\%) referred dyspareunia, 5 of them reported such symptom already before surgery; thus the de-novo dyspareunia rate was $17.6 \%$ ( 6 patients) at 12 months. At May 2016, 7 (20.6\%) out of 29 sexually active patients complained of dyspareunia, 4 of them were already affected before surgery. The de-novo dyspareunia rate at May 2016 was $10.3 \%$. At examination, the patients with de-novo dyspareunia presented vaginal dryness and/or vaginal rigidity. 


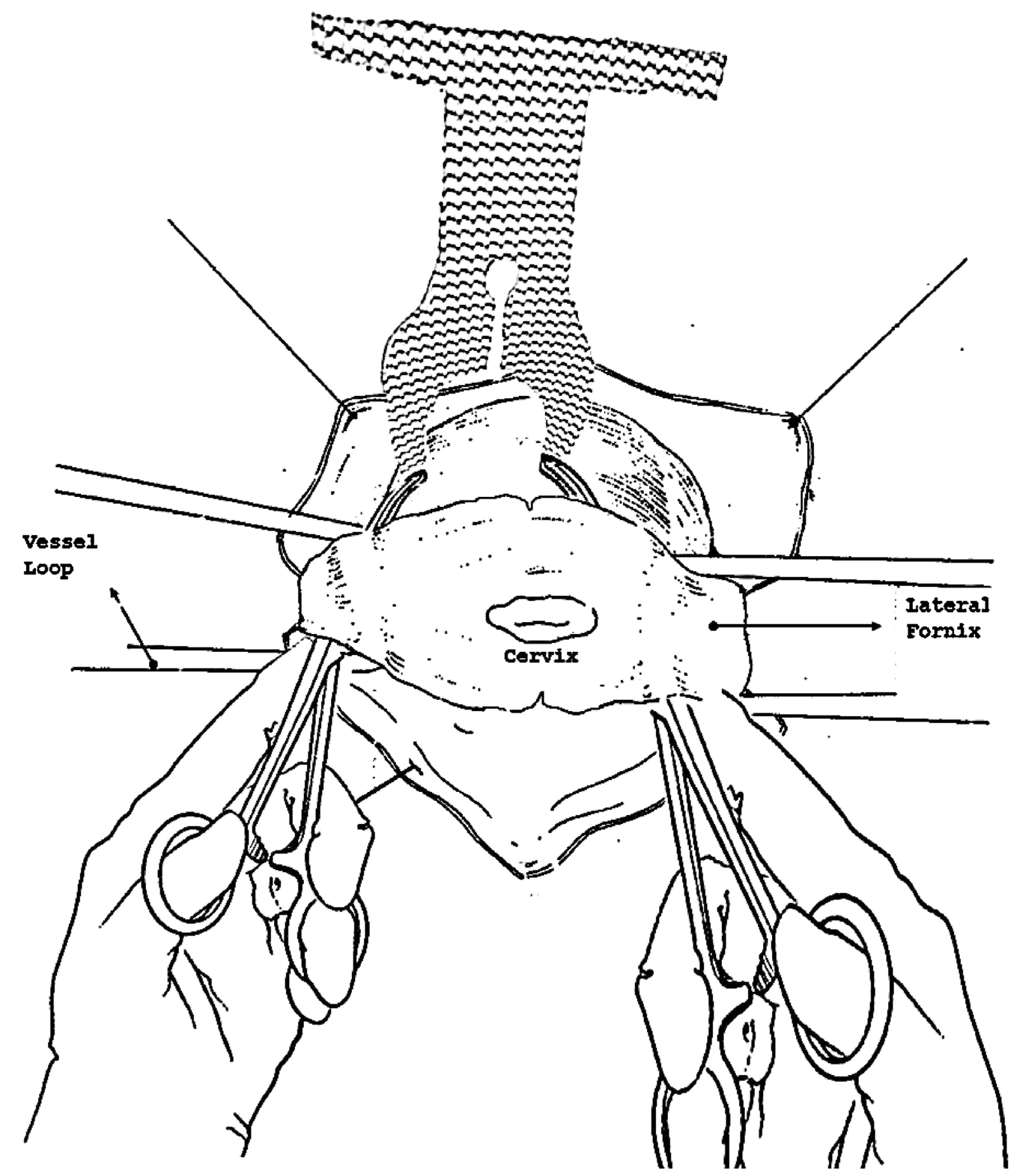

Fig. 2. Passage of the mesh between the posterior and anterior vaginal segments.

\section{Discussion}

Surgery aimed at pelvic floor reconstruction allows for different approaches: vaginal, open abdominal, laparoscopic or robotic. Operative time is generally shorter when the vaginal approach is adopted, as are hospital stay and recovery time [13,14]. Biocompatible or synthetic materials can be employed in pelvic floor reconstructive surgery, the debate about the best option is ongoing $[15,16]$. Synthetic meshes have been highly criticized in recent years, but the use of mesh in pelvic floor surgery is the reconstructive technique that best corresponds to anatomy because the shape of the mesh correctly reproduces the human pelvic floor. Moreover literature has underlined the uterus sparing technique as a viable option for the surgical management of uterine prolapse, because it is best at retaining the original anatomy and statics of the pelvic floor [17].

The results of our study confirm the effectiveness of our uterussparing technique for the correction of high-grade anterior and 


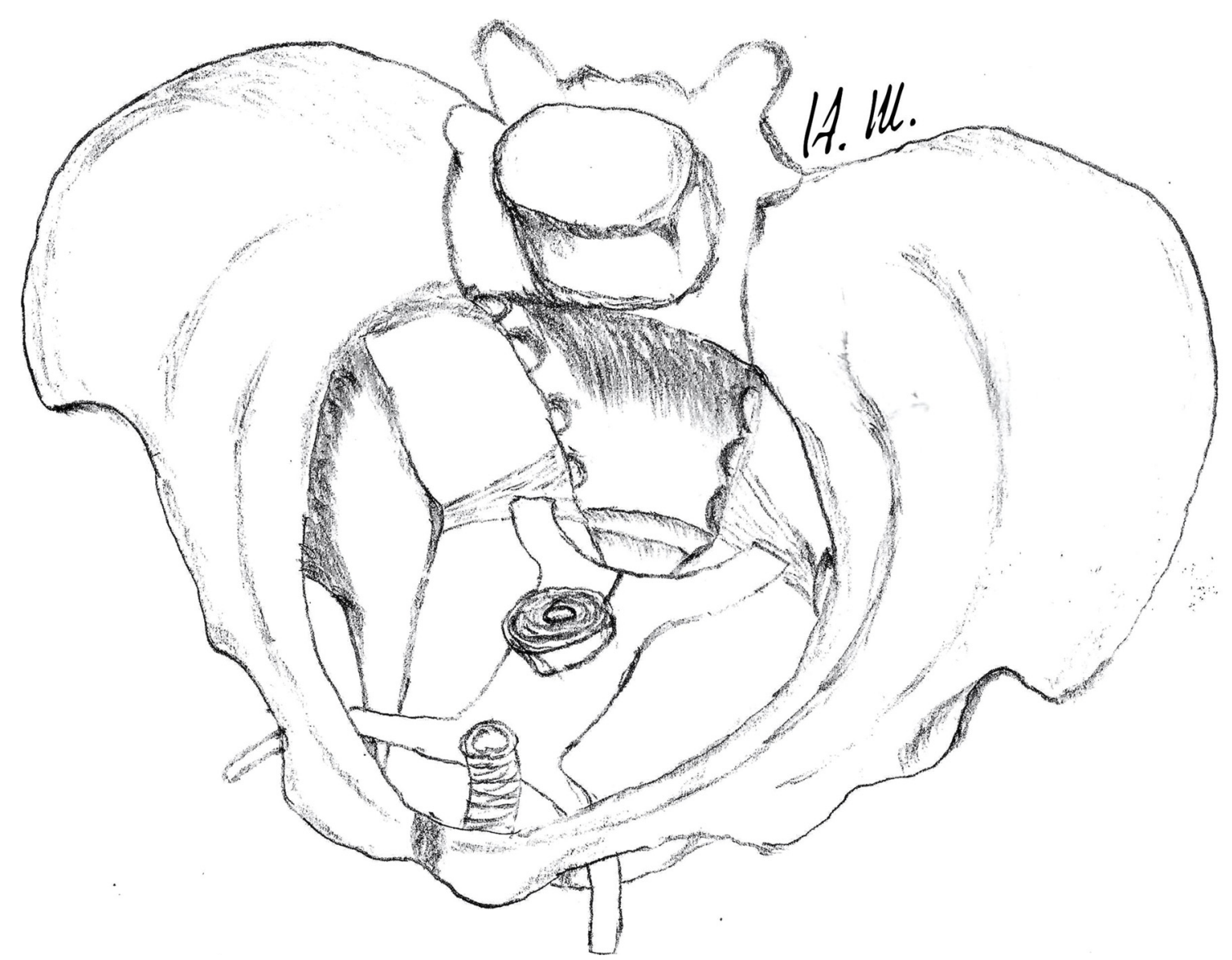

Fig. 3. Mesh in the pelvis. Upper view.

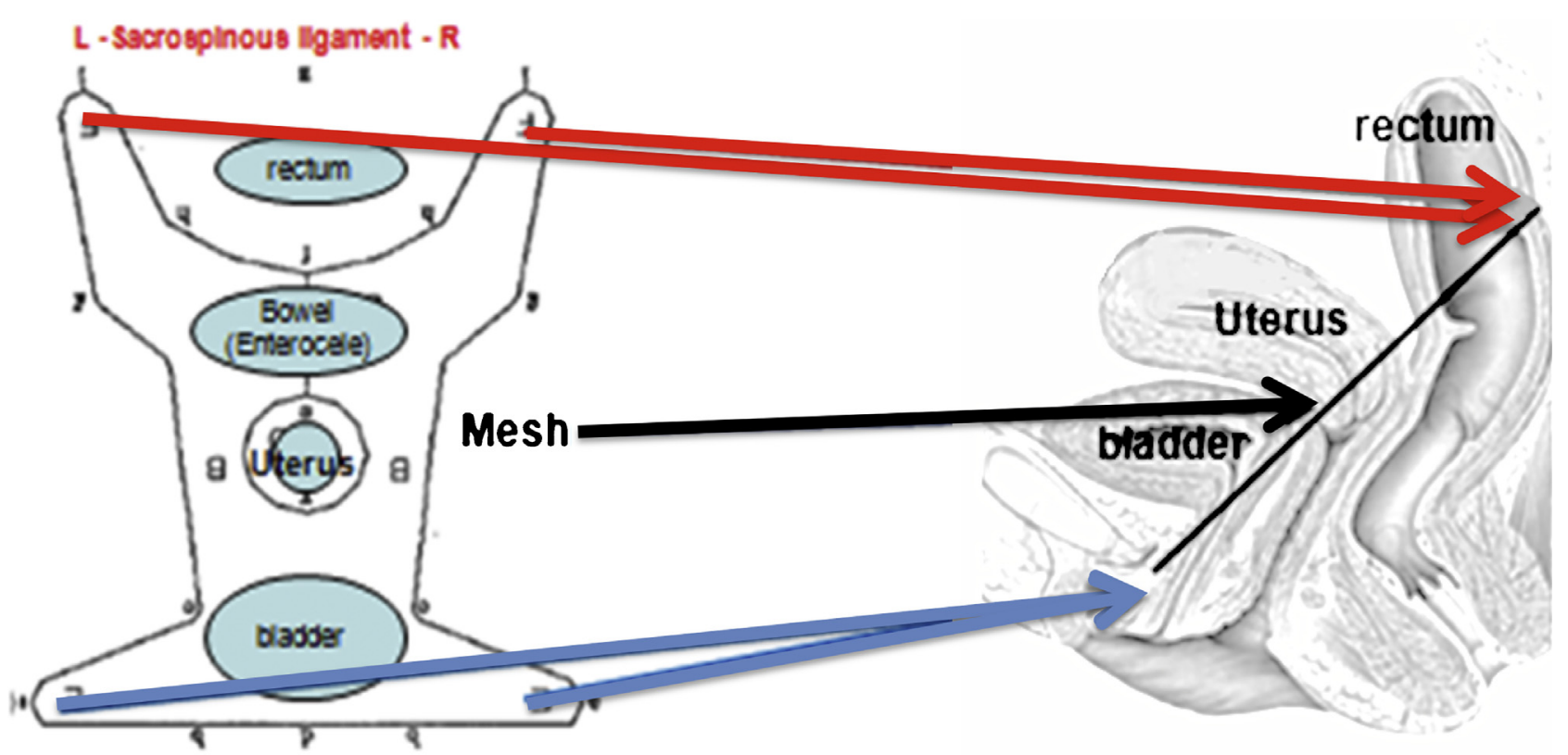

L. Wingo paseod traneobturator $R$

Fig. 4. Mesh in the pelvis. Schematic lateral view.

apical defects in terms of anatomical correction. Our rate of success is comparable to data reported in the literature for different procedures $[1,18,19]$. The length of our follow-up period suggests that results are stable over time. We have also observed a significant improvement in prolapse symptoms and quality of life. Our positive results are due to the logic of the mesh, its shape, and 
Table 2

Data from POP-Q at different follow-up times.

\begin{tabular}{|c|c|c|c|c|}
\hline & Preoperative, $\mathrm{n}=66$ & 3 months postoperative, $n=66$ & 12 months postoperative, $\mathrm{n}=66$ & May $2016^{\mathrm{a}}, \mathrm{n}=64^{\mathrm{b}}$ \\
\hline Stage $0, \mathrm{n}(\%)$ & - & $10(15.1)$ & $9(13.6)$ & $7(11.3)$ \\
\hline Stage I, n (\%) & - & $32(48.5)$ & $33(50)$ & $31(50)$ \\
\hline Stage II, n (\%) & - & $19(28.9)$ & 19 (28.9) & $16(25.8)$ \\
\hline Stage III, n (\%) & $32(48.5)$ & $2(3)$ & $2(3)$ & $5(8.1)$ \\
\hline Stage IV, n (\%) & $34(51.5)$ & $3(4.5)$ & $3(4.5)$ & $3(4.8)$ \\
\hline
\end{tabular}

a 2 patients were lost at May 2016 follow-up: one who had Stage IV POPQ at 12 months and another who had Stage II at 12 months.

b 2 patients underwent hysterectomy in another hospital, after 2 and 4 years of surgery respectively and have been considered as unsuccesful repair.

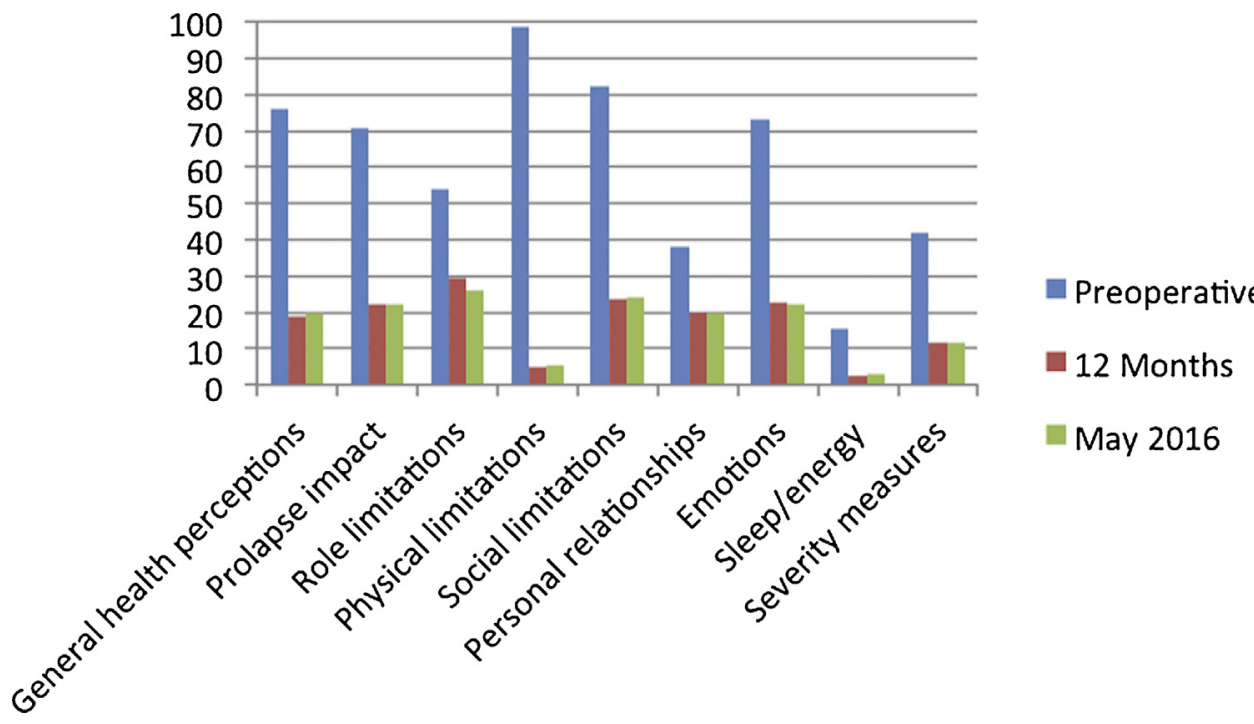

Fig. 5. Data from $P$ - QoL.

also its correct implementation. The pubo-cervical fascia, interposed between mesh and bladder acts as a buffer avoiding direct contact between bladder and mesh. We prefer to interpose the fascia between the mesh and the bladder to add protection to this organ. The rare vesical erosions are much more serious than those of the vagina, which are easily repaired.

Moreover when the pubocervical fascia is isolated from the bladder and left attached to the vaginal wall, the weak site (where erosions often occurred), is exactly where the walls and fascia are cut and then sutured. The procedure counteracts the causes of prolapse by recreating a new pelvic floor support, and implies no organ removal. The mesh, ultimately lies on a plane with the posterior part higher than the anterior one, thus it reestablishes the anatomical floor of the levator ani muscle, it is placed tensionfree in the pelvis, avoiding wrinkles that are the primary cause of vaginal erosions. The rectum, located between the posterior mesh wings, maintains its anatomical position and is under no pressure from the mesh.

Surgical correction of uterovaginal prolapse traditionally involves hysterectomy even if the uterus is believed to be passive in the disease. Removal of the uterus disrupts the equilibrium between the interactions of the pelvic organs regarding organ descent [20]. Additionally, hysterectomy cures the main "prolapse symptom", but it is less efficient in treating the cause, which is weakened support from musculofascial structures [21]. The void created by hysterectomy can cause a weakened area through which vaginal segments may prolapse [22].

Over the past few decades, uterus-sparing surgery, which we were among the first to practice [3], has become more popular worldwide and there is growing consensus that the uterus can be preserved in pelvic reconstructive surgery in appropriate patients
$[23,24]$. In fact, when hysterectomy is associated with POP surgery, operation time grows, blood loss is greater, and there are generally more complications, above all when a mesh is inserted by a vaginal approach [24]. Moreover, there is debate about whether anatomical modifications induced by hysterectomy implicate sexuality [25].

The use of meshes in treating POP was prompted by their use in incontinence surgery: the ease of implementation and positive results obtained suggested that meshes might yield similar results in POP surgery. Many products appeared on the market without appropriate clinical trials due to their simple production technology and high profit margins [26]. Furthermore, surgeons with little experience in this field were attracted by the apparent simplicity of use offered by commercial kits, some of them were difficult to use due to dimensions and anchoring technique. All these factors are responsible for a great amount of complications in patients receiving trans-vaginal procedures, leading to lawsuits failed against surgeons and industries. These circumstances led the FDA to require strict pre-clinical validation of meshes, and to a reclassification of these devices. Now they must meet stricter standards of efficacy for resolving the anatomical problem they intend to correct before they reach the market. In addition, a focused training which, however, has not yet been standardized, should be required. Indeed surgical experience is of pivotal importance [1,27]. Kelly et al. found the risk of complications to be lower for surgeons performing 14 or more procedures per year [27]. We agree with the FDA restrictions on the use of meshes for POP, but we take issue on their designation as high risk devices [2]. A specific risk resulting from the use of mesh in pelvic floor reconstruction surgery is mesh exposure [26]. The modern soft polypropylene large weave meshes reduced this risk as our low 
rate and grade of minor vaginal erosions demonstrate. Other risks such as dyspareunia and pelvic floor pain may also result from biocompatible materials [28]. Dyspareunia in post-menopausal women is not uncommon even without POP, its entity is usually difficult to evaluate. The rate of de-novo dyspareunia among our sexually active patients was $10.3 \%$, which is comparable to values reported by other authors [15,29], none of our patients referred pelvic pain attributable to sacrospinous ligaments stitches.

Despite criticism of synthetic materials, recent Cochrane reviews, albeit with metanalyses biases, affirmed that the use of vaginal meshes lowers the risk of prolapse symptoms and of recurrent anterior vaginal prolapse when compared to biocompatible tissue and fascial repair [26,30]. Our results are in keeping with those findings, confirming stable success rate over the longterm.

The limits of this study are: its monocentric prospective observational structure with a limited number of patients and its lack of a comparison with other techniques, such as hysterectomy and fascial repair. Uterus sparing surgery does raise questions of most suitable approach, whether vaginal, abdominal, or laparoscopic.

In conclusion, our technique is feasible for a urogynecologist of average experience trained by taking a hand in a few procedures. The low rate of complications demonstrates the safety both of the material and the procedure, which offers a stable and notable anatomical correction of prolapse with improvements in patient QoL. This finding warrants prospective evaluation in clinical trials. More experience is needed to determine which approach is best for prolapse surgery.

\section{Financial disclaimer/conflict of interest}

None.

\section{Aknowledgement}

To Prof. Gail Solberg for linguistic revisions, with thanks.

\section{References}

[1] Patel DN, Anger JT. Surgery for pelvic organ prolapse. Curr Opin Urol 2016;26:302-8.

[2] FDA Safety Communication. Update on serious complications associated with transvaginal placement of surgical mesh for pelvic organ prolapse. 2013. http://www.fda.gov/medicaldevices/safety/alertsandnotices/publichealthnotifications/ucm061976.htm.

[3] Nicita G. A new operation for genitourinary prolapse. J Urol 1998;160:741-5.

[4] Nicita G, Li Marzi V, Filocamo MT, et al. Uterus-sparing vaginal surgery of genitourinary prolapse employing biocompatible material. Urol Int 2005;75:314-8.

[5] Amid PK, Lichtenstein IL. Current assessment of Lichtenstein tension-free hernia repair. Chirurg 1997;68:959-64 [Article in German].

[6] DeLancey JO. Anatomic aspects of vaginal eversion after hysterectomy. Am ] Obstet Gynecol 1992;166:1717.

[7] Ingelman-Sundberg A, Ulmsten U. Surgical treatment of female urinary stress incontinence. Contrib Gynecol Obstet 1983;10:51-69.
[8] Schafer W, Abrams P, Liao L. Good urodynamic practices: uro-flowmetry, filling cystometry and pressure flow studies. Neurourol Urodyn 2002;21:261-74.

[9] Digesu GA, Santamato S, Khullar V, et al. Validation of an Italian version of the prolapse quality of life questionnaire. Eur J Obstetrics Gynecology Reprod Biol 2003;106:184-92.

[10] Dindo D, Demartines N, Clavien PA. Classification of surgical complications: a new proposal with evaluation in a cohort of 6336 patients and results of a survey. Ann Surg 2004;240:205-13.

[11] Miklos JR, Chinthakanan O, Moore RD, et al. The IUGA/ICS classification of synthetic mesh complications in female pelvic floor reconstructive surgery: a multicenter study. Int Urogynecology J 2016;27:933-8.

[12] Haylen BT, Freeman RM, Swift SE, et al. An international urogynecological association (IUGA)/international continence 284 society (ICS) joint terminology and classification of the complications related directly to the insertion of prostheses (meshes, implants, tapes) \& grafts in female pelvic floor surgery. Neurourol Urodynamics 2011;30:2-12.

[13] Morley GW, DeLancey JO. Sacrospinous ligament fixation for eversion of the vagina. Am J Obstetrics Gynecology 1988;158:872-81.

[14] Shull BL. Pelvic organ prolapse: anterior, superior, and posterior vaginal segment defects. Am J Obstetrics Gynecology 1999;181(July):6-11.

[15] McCammon K. Mesh rather than autologous tissue should be used for transvaginal repair of pelvic organ prolapse. Pro for mesh. J Urol 2016;195:251-2.

[16] Kobashi KC. Mesh rather than autologous tissue should be used for transvaginal repair of pelvic organ prolapse. Pro for native tissue. J Urol 2016;195:252-3.

[17] Gutman R, Maher C. Uterine-preserving POP surgery. Int Urogynecology J 2013;24:1803-13.

[18] Heinonen P, Aaltonen R, Joronen K, Ala-Nissilä S. Long-term outcome after transvaginal mesh repair of pelvic organ prolapse. Int Urogynecology J 2016;27 (July):1069-74.

[19] Siff LN, Barber MD. Native tissue prolapse repairs: comparative effectiveness trials. Obstetrics Gynecology Clinics North Am 2016;43:69-81.

[20] Fujiwara T, Togashi K, Yamaoka T, et al. Kinematics of the uterus: cine mode MR imaging. Radiographics 2004;24:e19.

[21] Selcuk S, Cam C, Asoglu MR, et al. Effect of simple and radical hysterectomy on quality of life - analysis of all aspects of pelvic floor dysfunction. Eur J Obstetrics Gynecology Reprod Biol 2016;198:84-8.

[22] Rappa C, Saccone G. Recurrence of vaginal prolapse after total vaginal hysterectomy with concurrent vaginal uterosacral ligament suspension: comparison between normal-weight and overweight women. Am J Obstetrics Gynecology 2016(June).

[23] Ridgeway BM. Does prolapse equal hysterectomy: the role of uterine conservation in women with uterovaginal prolapse. Am J Obstetrics Gynecology 2015;213:802-9.

[24] Cayrac M, Warembourg S, Le Normand L, Fatton B. Does hysterectomy modifies the anatomical and functional outcomes of prolapse surgery? Clinical practice guidelines. Prog Urol 2016;26(Suppl. 1)S73-88 [Article in French].

[25] Kokcu A, Kurtoglu E, Bildircin D, Celik H, Kaya A, Alper T. Does surgical menopause affect sexual performance differently from natural menopause. J Sex Med 2015;12:1407-14

[26] Maher C, Feiner B, Baessler K, Schmid C. Surgical management of pelvic organ prolapse in women. Cochrane Database Syst Rev 2016;4:CD004014, doi:http:// dx.doi.org/10.1002/14651858.CD004014.pub5.

[27] Kelly EC, Winick-Ng J, Welk B. Surgeon experience and complications of transvaginal prolapse mesh. Obstetrics Gynecology 2016;128:65-72.

[28] Farthmann J, Mengel M, Henne B, et al. Improvement of pelvic floor-related quality of life and sexual function after vaginal mesh implantation for cystocele: primary endpoint of a prospective multicentre trial. Arch Gynecology Obstetrics 2016;294:115-21.

[29] Gupta P, Payne J, Killinger KA, et al. Analysis of changes in sexual function in women undergoing pelvic organ prolapse repair with abdominal or vaginal approaches. Int Urogynecology J 2016(June).

[30] Maher C, Feiner B, Baessler K, Christmann-Schmid C, Haya N, Marjoribanks J. Transvaginal mesh or grafts compared with native tissue repair for vaginal prolapse. Cochrane Database Syst Rev 2016;9(February (2)):CD012079, doi: http://dx.doi.org/10.1002/14651858.CD012079. 